\title{
What Role Does Collaboration across Disciplines have in Responding to COVID-19?
}

\author{
jimi adams $\& 3$ Ryan Light
}

March 20, 2020

As the severe acute respiratory syndrome coronavirus 2 (SARS-COV-2) - the virus that causes COVID-19spreads across the globe, scientific coordination plays a key role in developing an effective response, just as in any rapidly unfolding event. This need for an appropriate but speedy response can run counter to scientific norms that are often seen as deliberate (slow), cautious (desiring abundance of evidence before recommending actions), and conservative (not prone to change).

The consequences of this pandemic and our efforts to curb its effects are profoundly social. These efforts involve the coordination of large communities of scientists, policy makers, and citizens. Therefore, it is important to understand the social dimensions that provide the backbone of those efforts, and theoretical and empirical tools for analyzing the structure and changes of scientific fields can helpfully inform them. Thus, here we: (1) describe the intersection of a sampling of the disciplinary perspectives needed to develop an effective response, (2) illustrate how this has translated into coordinated COVID-19 responses to date, and (3) discuss the potential of "open science" in these efforts.

\section{Why Collaboration Matters}

A key premise of the push for interdisciplinary research is that integrative scientific approaches draw on key insights from divergent disciplinary perspectives when addressing new problems, in ways that can lead to more rapid resolution. This implies that any efforts to effectively and quickly curtail the potentially devastating effects of COVID-19 necessitate drawing together the vast array of applicable scientific resources.

Scientists mobilize resources to solve major challenges often through collaboration. For example, developing accurate testing kits involves the expertise of biochemists and virologists. Distributing these kits enlists those with knowledge of how to optimize production and supply chains; social/behavioral scientists' knowledge shapes how to optimize roll-out, and testing administration and assessing results involves clinicians and lab techs from a range of specialties.

Accurate surveillance data are a backbone for developing intervention efforts, which variously combine the skill-sets of statisticians, epidemiologists, and demographers. These efforts inform the modeling efforts conducted by others with complementary skill-sets in those and related fields. Any such projections necessarily incorporate substantial amounts of uncertainty, given how knowledge rapidly changes.

Complexity science demonstrates how small behavioral changes or virologic differences can produce huge alterations to expected trajectories, allowing the range of plausibility to incorporate even the best- and worst-case scenarios. This uncertainty requires effective science communication skills to avoid under- or overreacting based on any single model, with computer, information, and political sciences needed to prevent the spread of misinformation. Moreover, model assemblages-aggregating across multiple existing strategies-are necessary to justify optimal response strategies using the best current information. Survey researchers from across the social sciences, combined with statisticians, have the tools to assess how prevalence estimates' sampling biases according to symptomatology require adjustment to incorporate with epidemiologists' and demographers' calculations of case fatality rates from those estimates.

Any hope for a vaccine requires the best work from immunologists, geneticists, and many of the other groups listed above. Psychologists, sociologists, and economists will be needed to understand the downstream consequences for mental health, social relations, and economic impacts. In sum, each individual task necessitates interdisciplinary integration, and the collective response is also a multi-level integration effort. 

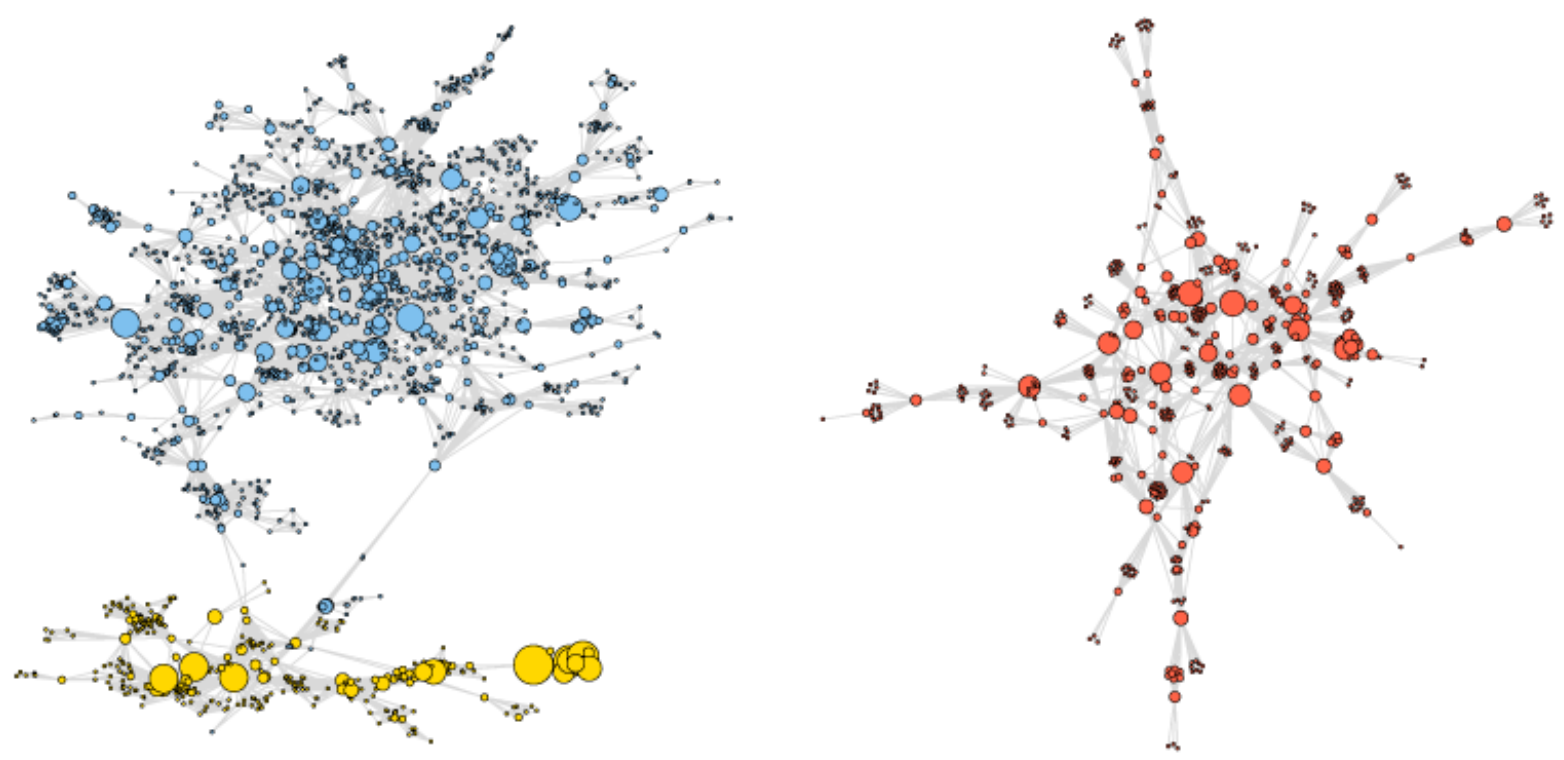

Figure 1: Figure 1. COVID-19 Collaboration Network. Network constructed from 1,070 articles published on COVID-19 between December 2019 and March 18, 2020 indexed by the PubMed database. Nodes consist of scientists and the node size is based on the number of articles authored by the scientist within this collection. Node color indicates a network community as determined by edge betweenness. Edges are based on coauthorship.

\section{What's happened so far}

In a little over three months, the scientific community has already developed a wide-ranging approach to understanding COVID-19, reflecting these interdisciplinary, collaborative efforts.

To demonstrate this, consider the 1,070 publications included in the PubMed MESH term for COVID-19 between December 2019 and March 18, 2020, remarkable in itself. From these articles, we constructed a coauthorship network (where each node represents a person, and each tie represents having written a paper together). Collaboration within this community is already robust; of the 5,111 authors, 5,006 have collaborated with at least one other author. Furthermore, the two large connected "components" (illustrated in Figure 1) include 2230 and 564 (red in Figure 1) scientists, respectively. The large component on the leftconsists of two clusters of $(1,864$, blue and 366, yellow) scientists loosely held together by a handful of collaborations.

Next, we asked whether collaboration is related to geographic and specialization distributions by examining first authors' addresses (where available) and publications' keywords. Geographic region plays an important role in structuring scientific collaboration. The blue cluster consists of scientists from across the globe, but 


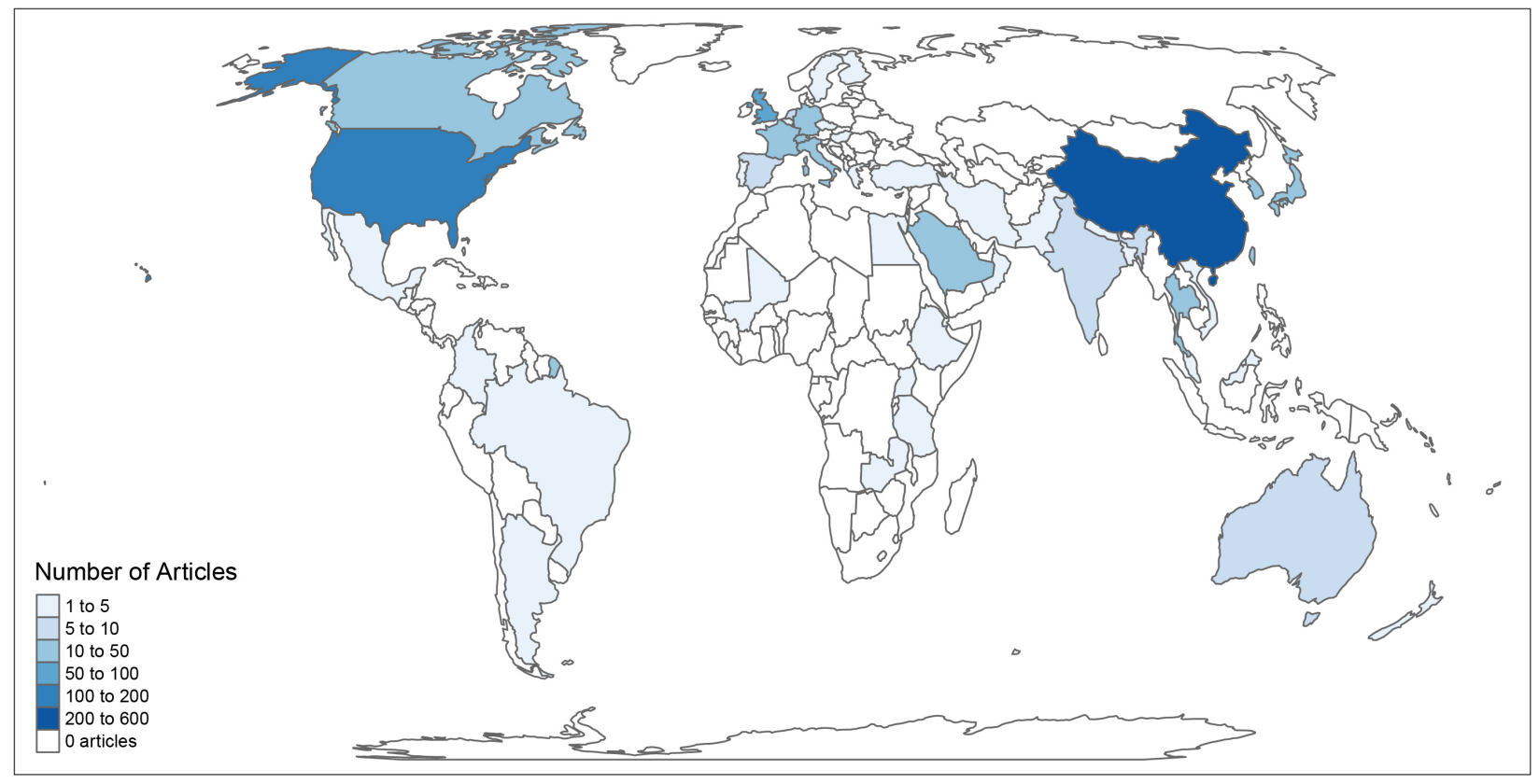

Figure 2: Figure 2. Map of COVID-19 Science. This map illustrates the 52 countries represented among the first authors of these COVID-19 articles. This is likely a conservative picture of the participation of the global scientific community.

disproportionately includes authors from China and the U.S. The yellow cluster is also diverse but has the largest representation from Japanese authors. The red cluster almost exclusively consists of scientists located in China. Of additional note, while researchers from the UK and Italy are actively involved, they are more likely to be in the smaller, disconnected components not represented in Figure 1.

Research specialization at this stage is more integrated with scientists from many fields tackling the core initial questions about the biology and epidemiology of COVID-19 and, therefore, substantive distinction is less pronounced. The top keyword in each group is "pneumonia" or "SARS." However, there is evidence of some subtle variation, with scientists in the blue cluster more likely to be working on diagnostic and genetic questions around keywords like "genome, viral" and "radiography, thoracic," while the wider network is more likely to be working on epidemiological questions associated with keywords like "epidemiology" and "outbreak."

The rapidly emerging science of COVID-19 is truly a global effort. Figure 2 illustrates that over 50 countries are represented among the publications' first authors. Several countries (China, the U.S., and Japan in particular) are producing sizable proportions of this research, but the distribution across the globe highlights impressive and widespread scientific mobilization.

The ability to mobilize and coordinate quickly and broadly likely reflects the extent of COVID-19's potential consequences. But it also has built upon the scientific community's increasing embrace of a number of principles of open science, and corresponding efforts to develop infrastructure that facilitates data and information sharing. In particular, prominent examples of this can be seen in NextStrain, Open-COVID, and the CORD-19 challenge.

\section{What have we learned \& where do we go from here?}

While there are many uncertainties about the nature of a new pathogen and the ensuing COVID-19 pandemic, scientific mobilization that integrates knowledge from across the wide ranging intersection of applicable expertise will optimize our response strategy. Early rapid mobilization seems to be building on these potentialities. As we continue to coordinate these efforts, sociological contributions can continue to identify 
opportunities for improved social coordination, even while practicing social distancing.

\section{Additional Resources}

- Garret Christensen, Jeremy Freese, \& Edward Miguel. 2019. Transparent and Reproducible Social Science Research: How to Do Open Science. University of Califronia Press.

- Ryan Light \& jimi adams. 2016. "A Dynamic, Multidimensional Approach to Knowledge Production." Chapter 6 in Scott Frickel, Mathieu Albert \& Barbara Prainsack (eds.) Investigating Interdisciplinary Collaboration: Theory \& Practice Across the Disciplines. Rutgers University Press.

All replication code is available from https://github.com/jimiadams/COVID_PubMed_Public. 